\title{
Duality for Semi-Definite and Semi-Infinite Programming ${ }^{1}$
}

\author{
S. J. LI \\ Department of Information and Computer Sciences, College of Sciences, \\ Chongqing University, Chongqing, 400044, China. \\ E-mail: 00900470r@polyu.edu.hk \\ X. Q. Yang And K. L. TeO \\ Department of Applied Mathematics, The Hong Kong Polytechnic University, \\ Kowloon, Hong Kong \\ E-mail: mayangxq@polyu.edu.hk and mateokl@polyu.edu.hk.
}

\begin{abstract}
In this paper, we study semi-definite and semi-infinite programming problems (SDSIP), which includes semi-infinite linear programs and semi-definite programs as special cases. We establish that a uniform duality between the homogeneous (SDSIP) and its Lagrangian-type dual problem is equivalent to the closedness condition of certain cone. Moreover, this closedness condition was assured by a generalized canonically closedness condition and a Slater condition. Corresponding results for the nonhomogeneous (SDSIP) problem were obtained by transforming it into an equivalent homogeneous (SDSIP) problem.
\end{abstract}

Keywords: Duality, semi-definite program, semi-infinite program.

\footnotetext{
${ }^{1}$ This research is partially supported by the Research Committee of The Hong Kong Polytechnic University and the National Natural Science Foundation of China.
} 


\section{Introduction}

Let $S^{n}$ denote the set of real symmetric $n \times n$ matrices. The standard inner product on $S^{n}$ is

$$
A \bullet B=\operatorname{tr} A B=\sum_{i, j} a_{i j} b_{i j}
$$

We consider the following semi-definite and semi-infinite linear programming problem (SDSIP):

$$
\begin{array}{ll}
\text { inf } & C \bullet X \\
\text { s.t } & A(t) \bullet X \geq b(t), \quad t \in B, \\
& X \succeq 0
\end{array}
$$

Here $B$ is a compact set in $R, C$ and $A(t)(t \in B)$ are all fixed matrices in $S^{n}$, $b(t) \in R(t \in B)$ and the unknown variable $X$ also lies in $S^{n}$.

Example 1.1 If $B$ is a finite set, problem (SDSIP) becomes a general semi-definite programming problem (SDP):

$$
\begin{array}{ll}
\text { inf } & C \bullet X \\
\text { S.t. } & A_{i} \bullet X \geq b_{i}, \quad i=1,2, \cdots, m, \\
& X \succeq 0,
\end{array}
$$

see $[7]$ and $[9]$.

Example 1.2 If

$$
C=\left(\begin{array}{ccc}
c_{1} & & 0 \\
& \ddots & \\
0 & & c_{n}
\end{array}\right), A(t)=\left(\begin{array}{ccc}
a_{1}(t) & & 0 \\
& \ddots & \\
0 & & a_{n}(t)
\end{array}\right), \forall t \in B .
$$

and $x=\left(x_{1}, x_{2}, \cdots, x_{n}\right)^{T}$, (SDSIP) problem is reduced to a semi-infinite linear programming problem (SILP):

$$
\begin{array}{ll}
\text { inf } & c^{T} x \\
\text { s.t } & (a(t))^{T} x \geq b(t), \quad t \in B, \\
& x \geq 0,
\end{array}
$$


where $c=\left(c_{1}, c_{2}, \cdots, c_{n}\right)^{T}$ and $a(t)=\left(a_{1}(t), a_{2}(t), \cdots, a_{n}(t)\right)^{T}$. See [4].

Obviously, if a point $x^{*} \in R^{n}$ is a solution of (SILP), then the point

$$
X^{*}=\left(\begin{array}{ccc}
x_{1}^{*} & & 0 \\
& \ddots & \\
0 & & x_{n}^{*}
\end{array}\right) \in S^{n}
$$

is a solution of (SDSIP). Conversely, if a point $X^{*} \in S^{n}$ is a solution of (SDSIP), the point $x^{*}=\left(x_{11}, x_{22}, \ldots, x_{n n}\right)^{T} \in R^{n}$ is also a solution of (SILP).

Example 1.3 We consider a quadratically constrained semi-infinite quadratic programming problem $\left(Q^{2} P\right)$ :

$$
\begin{array}{ll}
\min & x^{T} Q_{0} x+2 g_{0}^{T} x+\alpha_{0} \\
\text { s.t. } & x^{T} Q(t) x+2 g(t)^{T} x+\alpha(t) \geq 0, t \in B,
\end{array}
$$

where $B$ is a compact set in $R$ and, $C, Q_{0}$ and $Q(t),(t \in B)$ are all symmetric matrices in $S^{n}$. Note that the objective function and the constraints are not necessarily convex. Therefore the feasible set of $\left(Q^{2} P\right)$ can be a very "nasty" set. This problem is, in general, very hard to solve, see e.g. [10].

Let

$$
P_{0}=\left[\begin{array}{ll}
\alpha_{0} & g_{0}^{T} \\
g_{0} & Q_{0}
\end{array}\right]
$$

and

$$
P(t)=\left[\begin{array}{ll}
\alpha(t) & g(t)^{T} \\
g(t) & Q(t)
\end{array}\right] .
$$

Then, an equivalent homogenized formulation to $\left(Q^{2} P\right)_{y}$ is

$$
\begin{array}{ll}
\min & y^{T} P_{0} y \\
\text { s.t. } & y^{T} P(t) y \geq 0, t \in B, \\
& y_{0}^{2}=1 \\
& y=\left(y_{0}, x^{T}\right)^{T} \in R^{n+1} .
\end{array}
$$

It is clear that if $\left(1,\left(x^{*}\right)^{T}\right)^{T}$ is optimal to $\left(Q^{2} P\right)_{y}$, then $x^{*}$ is optimal to $\left(Q^{2} P\right)$ and the values of $\left(Q^{2} P\right)$ at $x^{*}$ and $\left(Q^{2} P\right)_{y}$ at $\left(1,\left(x^{*}\right)^{T}\right)^{T}$ are equal. Since the values 
of $\left(Q^{2} P\right)_{y}$ at $\left(1,\left(x^{*}\right)^{T}\right)^{T}$ and $\left(-1,-\left(x^{*}\right)^{T}\right)^{T}$ are equal, we may consider that the optimal values of $\left(Q^{2} P\right)$ and $\left(Q^{2} P\right)_{y}$ are equal. The homogenization simplifies the notion and opens the way to the semidefinite relaxation since we can rewrite $\left(Q^{2} P\right)_{y}$ using matrix variables:

$$
\begin{array}{ll}
\min & P_{0} \bullet Y \\
\text { s.t. } & E_{00} \bullet Y=1, \\
& P(t) \bullet Y \geq 0, t \in B, \\
& \operatorname{rank}(Y)=1, \quad Y \succeq 0,
\end{array}
$$

where $E_{00}$ is the zero matrix with 1 in the top left corner. Dropping the $\operatorname{rank}(Y)=1$ provides a SDP relaxation of $\left(Q^{2} P\right)$ :

$$
\begin{array}{ll}
(\bar{P}) \quad \min & P_{0} \bullet Y \\
\text { s.t. } \quad & E_{00} \bullet Y=1, \\
& P(t) \bullet Y \geq 0, t \in B, \\
& Y \succeq 0 .
\end{array}
$$

Thus, problem (SDSIP) includes the semi-definite programming problem and the semi-infinite linear programming problem as special cases. Moreover, quadratically constrained semi-infinite quadratic programming problems can be transformed to problem (SDSIP). Conversely, it is well known that a semidefinite programming problem is a special case of a linear semi-infinite programming problem (see [8]). It is clear that the problem (SDSIP) also is a special linear semi-infinite programming problem.

It is well known that semi-definite programming (SDP) is an important research field. There are good reasons for studying (SDP). First, (SDP) problems directly arise in a number of important applications, e.g. structural optimization, discrete (combinatorial) optimization, systems and control problems. Second, many convex optimization problems, such as linear programming problems and convex quadratic programming problems, can be cast into semi-definite programming problems. Although (SDP) is a generalization of linear programming problems, the simplex 
method for linear programming cannot be generalized for solving (SDP) and the primal (SDP) and its dual (SDP) may have a nonzero duality gap. Wolkowicz et al [15] proved that there is a zero duality gap between the primal and dual (SDP) problems if a Slater constraint qualification condition holds. Ramana et al [11]-[12] introduced a kind of the Extended Lagrange-Slater dual problem (ELSD) for (SDP) and proved that there is a zero gap between the primal (SDP) and dual problem (ELSD) without any constraint qualification.

Duality theory for semi-infinite linear programming problem (SILP) is also an important research field (see [2], [3] and [13]). Researchers found that there is, in general, a nonzero duality gap between (SILP) and its dual problem. Duffin and Karlovitz [3] obtained a zero duality gap for (SILP) under a canonically closedness condition and a Slater condition. Duffin et al [2] investigated uniform duality for (SILP), i.e., a zero duality gap holds between (SILP) and its dual problem for any cost vector $c$.

Motivated by the work reported in [15] and [2], we study uniform duality for (SDSIP). First, we get a necessary and sufficient condition for the uniform duality of homogeneous (SDSIP) problem. Then, we transform a nonhomogeneous (SDSIP) problems into an equivalent homogeneous (SDSIP) problem. Under a generalized canonically closedness condition and a Slater condition we establish uniform duality property for nonhomogeneous (SDSIP) with the aid of the result of homogeneous (SDSIP) problem. Moreover, we show that a zero duality gap exists between (SDSIP) and its dual problem if a Slater condition holds.

The rest of the paper is organized as follows: In Section 2, Lagrangian dual problem for (SDSIP) is formulated and uniform duality of problem (SDSIP) is introduced. In Section 3, the uniform duality for homogeneous (SDSIP) problems is obtained. In Section 4, the uniform duality for nonhomogeneous (SDSIP) problems is discussed with aid of the results of homogeneous (SDSIP) problems. In Section 5 , the uniform duality for (SDSIP) problems is obtained under the Slater condition. 


\section{Uniform Duality for (SDSIP)}

Let us first introduce some notations. For the compact set $B$, Let $R^{B}=\prod_{B} R$ denote the product space in the product topology, which is a locally convex Hausdorff topological vector space; see [6]. Then, the topological dual space of $R^{B}$ is the generalized finite sequence space consisting of all functions $g: B \rightarrow R$ with finite support. The set $R_{+}^{B}=\prod_{B} R_{+}$denotes the convex cone of all nonnegative functions on $B$. Then, the dual cone of $R_{+}^{B}$ is the set

$$
\begin{aligned}
\Lambda_{B}=\quad & \left\{y=\{y(t)\}_{t \in B} \mid(\exists \text { a finite set } F \subseteq B)(\forall t \in B \backslash F) y(t)=0\right. \\
& \text { and }(\forall t \in F) y(t) \geq 0\} .
\end{aligned}
$$

We let

$$
\begin{aligned}
\Lambda_{B}^{1}=\quad & \left\{y=\{y(t)\}_{t \in B} \mid(\exists \text { a finite set } F \subseteq B)(\forall t \in B \backslash F) y(t)=0\right. \\
& \left.(\forall t \in F) y(t) \geq 0 \text { and } \sum_{t \in B} y(t)=1\right\} .
\end{aligned}
$$

See [5].

By $X \succeq 0$, where $X \in S^{n}$, we mean that the matrix $X$ is positive semidefinite. The set $K=\left\{X \in S^{n} \mid X \succeq 0\right\}$ is called the positive semidefinite cone. For any $S \subset S^{n}, \operatorname{cl}(S)$ denotes the closure of $S$ in $S^{n}$. For the set $W=\{A(t) \mid t \in B\}$, cone $(W)$ denotes the cone generated by $W$, i.e.,

$$
\operatorname{cone}(W)=\left\{\sum_{t \in B} y(t) A(t) \mid \forall y \in \Lambda_{B}\right\},
$$

and $\operatorname{conv}(W)$ denotes the convex hull generated by $W$, i.e.,

$$
\operatorname{conv}(W)=\left\{\sum_{t \in B} y(t) A(t) \mid \forall y \in \Lambda_{B}^{1}\right\},
$$

For semi-definite and semi-infinite programming problem (SDSIP), we introduce the Lagrangian dual problem (DSDSIP) as follows:

$$
\begin{array}{ll}
\sup & \sum_{t \in B} y(t) b(t) \\
\text { s.t } & \sum_{t \in B} y(t) A(t)+Z=C, y \in \Lambda_{B}, \\
& Z \succeq 0 .
\end{array}
$$


When the parameter set $B$ is finite, Then, (SDSIP) and (DSDSIP) is a pair of primal and dual (SDP). See [15].

The program (SDSIP) is said to be consistent if its constraint system (1) has a solution. It is said to be bounded in value if it is consistent and there exists a number $z^{*}$ such that all feasible solutions $X \in S^{n}$ to (SDSIP) satisfy $C \bullet X \geq z^{*}$. It is said to unbounded in value if, for each integer $n$, there exists a feasible solution $X^{(n)}$ to $(\mathrm{SDSIP})$ with $C \bullet X^{(n)} \leq-n$.

Definition 2.1 The system of linear equalities

$$
A(t) \bullet X \geq b(t), \quad t \in B
$$

yields duality with respect to $C \in S^{n}$, if exactly one of the following conditions holds:

(i) (SDSIP) is unbounded in value and (DSDSIP) is inconsistent;

(ii) (DSDSIP) is unbounded in value and (SDSIP) is inconsistent;

(iii) Both (SDSIP) and (DSDSIP) are inconsistent;

(iv) Both (SDSIP) and (DSDSIP) are consistent and have the same optimal value, and the value is attained in (DSDSIP).

We say that (SDSIP) yields uniform duality if the constraint system (3) yields duality for every $C \in S^{n}$.

Definition 2.2 $A(t)(t \in B)$ is said to be generalized canonically closed, if there exists a set of positive constants $k(t)(t \in B)$ such that the set $\{k(t) A(t) \mid t \in B\}$ forms a compact set in $S^{n}$.

Remark 2.1 Let

$$
\tilde{A}(t)=\left(\begin{array}{cccc}
a_{1}(t) & & & 0 \\
& \ddots & & \\
& & a_{n}(t) & \\
0 & & & -b(t)
\end{array}\right)(t \in B)
$$


be $(n+1) \times(n+1)$ diagonal matrices. Then, there exists a set of positive constants $k(t)(t \in B)$ such that the set $\{k(t) \tilde{A}(t) \mid t \in B\}$ forms a compact set in $S^{n+1}$ if and only if there exists a set of positive constants $k(t)(t \in B)$ such that the set $\left\{k(t)\left(a_{1}(t), \cdots, a_{n}(t), b(t)\right) \mid t \in B\right\}$ forms a compact set in $R^{n+1}$, i.e., the set $\left\{\left(a_{1}(t), \cdots, a_{n}(t), b(t)\right) \mid t \in B\right\}$ is canonically closed. The later is introduced by Charnes, Cooper and Kortanek [1]. Thus, Definition 2.2 is a generalization of the definition for canonically closed in [1].

Remark 2.2 If $b(t)=0, \forall t \in B$, it is clear that $\tilde{A}(t)=\left(\begin{array}{cc}A(t) & \mathbf{0} \\ \mathbf{0}^{T} & 0\end{array}\right)(t \in B)$ is generalized canonically closed if and only if $A(t)(t \in B)$ is generalized canonically closed.

\section{Homogeneous System}

In this section, we discuss the homogeneous case in (SDSIP): $b(t)=0, \forall t \in B$. Then (SDSIP) becomes the following problem $\left(\operatorname{SDSIP}_{h}\right)$ :

$$
\begin{array}{ll}
\text { inf } & C \bullet X \\
\text { s.t } & A(t) \bullet X \geq 0, t \in B, \\
& X \succeq 0
\end{array}
$$

and (DSDSIP) becomes the following problem $\left(\operatorname{DSDSIP}_{h}\right)$ :

$$
\begin{aligned}
& \sup 0 \\
& \text { s.t } \sum_{t \in B} y(t) A(t)+Z=C, y \in \Lambda_{B}, \\
& Z \succeq 0 .
\end{aligned}
$$

Lemma 3.1 The problem $\left(S D S I P_{h}\right)$ is unbounded in value if and only if there exists $X^{*} \succeq 0$ satisfying:

$$
\begin{aligned}
A(t) \bullet X^{*} & \geq 0, \quad t \in B \\
\text { and } \quad C \bullet X^{*} & <0 .
\end{aligned}
$$


Proof. Suppose that there is $X^{*} \succeq 0$ such that (6) and (7) hold. Without loss of generality, assume $C \bullet X^{*}<-1$. For each $n$ we have $A(t) \bullet X^{(n)} \geq 0, t \in B$ and $C \bullet X^{(n)}<-n$ with $X^{(n)}=n X^{*}$. Hence, $\left(\operatorname{SDSIP}_{h}\right)$ is unbounded in value.

Conversely, by the unbounded definition, the case holds. This completes the proof.

It is clear that $\left(\operatorname{SDSIP}_{h}\right)$ is always consistent as $X=0$ is a feasible solution. If the optimal value of $\left(\operatorname{SDSIP}_{h}\right)$ is bounded below, then by Lemma 3.1 we must have that the optimal value of $\left(\operatorname{SDSIP}_{h}\right)$ is zero. Thus, (ii) and (iii) in Definition 2.1 do not happen.

Theorem $3.1\left(S D S I P_{h}\right)$ yields uniform duality if and only if cone $(W)+K$ is a closed set.

Proof. Suppose $\left(\operatorname{SDSIP}_{h}\right)$ yields uniform duality. Let $C \in \operatorname{cl}(\operatorname{cone}(W)+K)$ and $C \notin \operatorname{cone}(W)+K$. Then, there exists no $y \in \Lambda_{B}$ and $Z \succeq 0$ such that

$$
\sum_{t \in B} y(t) A(t)+Z=C
$$

Thus, $\left(\operatorname{DSDSIP}_{h}\right)$ is inconsistent. Since $\left(\operatorname{SDSIP}_{h}\right)$ is consistent, the problem $\left(\operatorname{SDSIP}_{h}\right)$ must be unbounded in value. By Lemma 3.1, there exists $X^{*} \succeq 0$ satisfying (6) and (7). By (6), we have that

$$
V \bullet X^{*} \geq 0, \quad \forall V \in \operatorname{cone}(W)
$$

Take any $S \in \operatorname{cone}(W)+K$. Then, there exist $V \in \operatorname{cone}(W)$ and $Q \in K$ such that

$$
S=V+Q
$$

We have

$$
S \bullet X^{*}=V \bullet X^{*}+Q \bullet X^{*} \geq Q \bullet X^{*}
$$

Since $Q$ and $X^{*}$ are positive semidefinite matrices, we have that

$$
Q \bullet X^{*} \geq 0
$$

and

$$
S \bullet X^{*} \geq 0 \text {. }
$$


Therefore, we get that

$$
S \bullet X^{*} \geq 0, \quad \forall S \in \operatorname{cl}(\operatorname{cone}(W)+K),
$$

and

$$
C \bullet X^{*} \geq 0
$$

However, it follows from (7) that $C \bullet X^{*}<0$, which is a contradiction. Hence, cone $(W)+K$ is closed.

Conversely, suppose cone $(W)+K$ is closed. Let $C \in S^{n}$ be arbitrary. Since $\left(\mathrm{SDSIP}_{h}\right)$ is consistent, either $\left(\mathrm{SDSIP}_{h}\right)$ is unbounded in valued or bounded in value. If $\left(\mathrm{SDSIP}_{h}\right)$ is unbounded in value, $\left(\mathrm{DSDSIP}_{h}\right)$ is inconsistent. If $\left(\mathrm{SDSIP}_{h}\right)$ is bounded in value, its value is zero by Lemma 3.1. Now we show that clause (iv) of Definition 2.1 holds. If $\left(\operatorname{DSDSIP}_{h}\right)$ is not consistent for $C, C \notin \operatorname{cone}(W)+K=$ $c l(\operatorname{cone}(W)+K)$. By the definitions of cone $(W)$ and $K$, we have that cone $(W)+K$ is a closed and convex cone in $S^{n}$. Thus, by the separation theorem [14], there exists $X^{*}$ in $S^{n}$ such that

$$
C \bullet X^{*}<0 \text { and } V \bullet X^{*} \geq 0, \quad \forall V \in \operatorname{cone}(W)+K \text {. }
$$

Obviously,

$$
A(t) \in \operatorname{cone}(W)+K, \quad \forall t \in B
$$

Therefore,

$$
C \bullet X^{*}<0 \text { and } A(t) \bullet X^{*} \geq 0, \quad \forall t \in B
$$

Thus, it is necessary that we prove $X^{*} \succeq 0$. Take any $Q \in K$ and $0 \in \operatorname{cone}(W)$. We have

$$
Q \bullet X^{*} \geq 0, \quad \forall Q \in K
$$

If $X^{*}$ is not a positive semidefinite matrix, there exists $v \in R^{n}$ such that

$$
v^{T} X^{*} v<0
$$

Define: $Q=v v^{T}$. Thus, $Q$ is a positive semidefinite matrix. By (8),

$$
Q \bullet X^{*} \geq 0
$$


Nevertheless,

$$
Q \bullet X^{*}=\left(v v^{T}\right) \bullet X^{*}=v^{T} X^{*} v<0,
$$

which is a contradiction. Thus, $X^{*}$ is a positive semidefinite matrix. It completes this proof.

Now we give two examples to illustrate the theorem.

Example 3.1 Suppose that

$$
A(t)=\left(\begin{array}{cc}
-\sin (t) & 0 \\
0 & \cos (t)
\end{array}\right), t \in[0, \pi / 2], \text { and } C=\left(\begin{array}{cc}
-2 & -1 \\
-1 & 0
\end{array}\right) .
$$

The primal problem is defined as

$$
\begin{array}{ll}
\text { inf } & C \bullet X \\
\text { s.t } & A(t) \bullet X \geq 0, \quad t \in[0, \pi / 2], \\
& X \succeq 0 .
\end{array}
$$

The feasible set for the primal problem is:

$$
\mathcal{F}=\left\{\left(\begin{array}{ll}
0 & 0 \\
0 & x
\end{array}\right) \mid x \geq 0\right\}
$$

Then, an optimal solution for the primal problem is $X^{*}=\left(\begin{array}{ll}0 & 0 \\ 0 & 0\end{array}\right)$, and the optimal value is zero.

The dual problem of the primal problem is

$$
\begin{array}{ll}
\text { sup } & 0 \\
\text { s.t } & \sum_{t \in[0, \pi / 2]} y(t) A(t)+Z=C, y \in \Lambda_{[0, \pi / 2]}, \\
& Z \succeq 0 .
\end{array}
$$

However, the dual problem has no feasible solution, i.e., the dual problem is inconsistent. Thus, the primal problem does not yield uniform duality. Obviously, cone $\{A(t) \mid t \in[0, \pi / 2]\}+K=\left\{\left(\begin{array}{ll}x_{11} & x_{12} \\ x_{12} & x_{22}\end{array}\right) \mid x_{22} \geq 0\right.$ and $x_{22}>0$, when $\left.x_{12} \neq 0\right\}$, 
and

$$
c l(\operatorname{cone}\{A(t) \mid t \in[0, \pi / 2]\}+K)=\left\{\left(\begin{array}{ll}
x_{11} & x_{12} \\
x_{12} & x_{22}
\end{array}\right) \mid x_{22} \geq 0\right\} .
$$

Thus,

$$
\operatorname{cone}\{A(t) \mid t \in[0, \pi / 2]\}+K \neq \operatorname{cl}(\operatorname{cone}\{A(t) \mid t \in[0, \pi / 2]\}+K),
$$

i.e., the set cone $\{A(t) \mid t \in[0, \pi / 2]\}+K$ is not closed.

Example 3.2 Suppose that

$$
A(t)=\left(\begin{array}{cc}
t & 0 \\
0 & t
\end{array}\right), t \in[1,2] .
$$

For any $C \in S^{n}$, the primal problem is

$$
\begin{array}{ll}
\text { inf } & C \bullet X \\
\text { s.t } & A(t) \bullet X \geq 0, \quad t \in[1,2], \\
& X \succeq 0 .
\end{array}
$$

The feasible set for the primal problem is:

$$
\mathcal{F}=K
$$

If $C \succeq 0$, we can obtain that an optimal solution for the primal problem is $X^{*}=$ $\left(\begin{array}{ll}0 & 0 \\ 0 & 0\end{array}\right)$, and the optimal value is zero. If $C$ is not positive semidefinite, then, there is an $X \in \mathcal{F}$ such that

$$
C \bullet X<0 .
$$

Thus, the primal problem is unbounded in value.

The dual problem of the above problem is

$$
\begin{array}{ll}
\sup & 0 \\
\text { s.t } & \sum_{t \in[1,2]} y(t) A(t)+Z=C, y \in \Lambda_{[1,2]}, \\
& Z \succeq 0 .
\end{array}
$$

If $C \succeq 0$, then, the dual problem is consistent as $y=0$ and $Z=C$ is a feasible solution. 
By assumption of $A(t), t \in B$ and $Z \succeq 0$, we have that $\sum_{t \in[1,2]} y(t) A(t)+Z$ is a positive semidefinite matrix for any $y \in \Lambda_{B}$. Then, if $C$ is not positive semidefinite, we get that the dual problem is inconsistent. Thus, by Definition 2.1, the primal problem yields uniform duality. Obviously, we have

$$
\operatorname{cone}\{A(t) \mid t \in[1,2]\}+K=K \text {. }
$$

Therefore, cone $\{A(t) \mid t \in[1,2]\}+K$ is a closed set.

Next we consider a special case of Theorem 3.1, which will be used in the sequel. We assume that:

$$
\begin{gathered}
\bar{C}=\left(\begin{array}{cc}
C & \mathbf{0} \\
\mathbf{0}^{T} & c
\end{array}\right), \bar{A}(t)=\left(\begin{array}{cc}
A(t) & \mathbf{0} \\
\mathbf{0}^{T} & a(t)
\end{array}\right), t \in B, \\
\bar{W}=\{\bar{A}(t) \mid t \in B\}, \operatorname{cone}(\bar{W})=\left\{\sum_{t \in B} y(t) \bar{A}(t) \mid y \in \Lambda_{B}\right\},
\end{gathered}
$$

and

$$
\bar{K}=\left\{\left(\begin{array}{cc}
K & \mathbf{0} \\
\mathbf{0}^{T} & k
\end{array}\right) \in S^{n+1} \mid K \text { is positive semidefinite in } S^{n}, k \in R^{+}\right\}
$$

where $C, A(t) \in S^{n}, \mathbf{0}$ is zero element in $R^{n}$ and $c, a(t) \in R, \forall t \in B$. Let $\bar{X} \in S^{n+1}$. We can get the following result.

Proposition 3.1 The constraint system

$$
\bar{A}(t) \bullet \bar{X} \geq 0, t \in B
$$

yields duality for any $\bar{C} \in S^{n+1}$ if and only if cone $(\bar{W})+\bar{K}$ is a closed set.

Proof. The proof is similar to that of Theorem 3.1 and is omitted.

Remark 3.1 If cone $(\bar{W})+\bar{K}$ is closed, then the same happens with cone $(W)+K$, where cone $(W)=\left\{\sum_{t \in B} y(t) A(t) \mid y \in \Lambda_{B}\right\}$. Thus, if a system, as it is written in Proposition 3.1, yields uniform duality, then the corresponding reduced system:

$$
A(t) \bullet X \geq 0, t \in B
$$

also yields uniform duality, where $X \in S^{n}$. 


\section{Nonhomogeneous System}

We now establish the duality for the nonhomogeneous constraint system (1) of (SDSIP) by reformulating it as a form of homogeneous system (4) and applying Proposition 3.1. For any real number $d \in R$, we define:

$$
\tilde{C}=\left(\begin{array}{cc}
C & \mathbf{0} \\
\mathbf{0}^{T} & -d
\end{array}\right), \tilde{A}(t)=\left(\begin{array}{cc}
A(t) & \mathbf{0} \\
\mathbf{0}^{T} & -b(t)
\end{array}\right),
$$

$\mathbf{0}$ is zero element in $R^{n}$ and $\tilde{X}, \tilde{Z} \in S^{n+1}$.

Now, we introduce a new semi-definite and semi-infinite programming problem (SDSIP1):

$$
\begin{array}{ll}
\inf & \tilde{C} \bullet \tilde{X} \\
\text { s.t } & \tilde{A}(t) \bullet \tilde{X} \geq 0, \quad t \in B, \\
& \tilde{X} \succeq 0 .
\end{array}
$$

The Lagrangian dual program of (SDSIP1) is as follows:

$$
\begin{array}{ll}
\text { sup } & 0 \\
\text { s.t. } & \sum_{t \in B} y(t) \tilde{A}(t)+\tilde{Z}=\tilde{C}, \quad y \in \Lambda_{B}, \\
& \tilde{Z} \succeq 0 .
\end{array}
$$

which is equivalent to the program

$$
\begin{array}{ll}
\text { sup } & 0 \\
\text { s.t. } & \sum_{t \in B} y(t) A(t)+Z=C, \quad y \in \Lambda_{B}, \\
& \sum_{t \in B} y(t) b(t) \geq d, y \in \Lambda_{B}, \\
& Z \succeq 0 .
\end{array}
$$

Lemma 4.1 The nonhomogeneous constraint system (1) yields duality with respect to $C \in S^{n}$ if and only if, for every $d \in R$, the constraint system (12) yields duality with respect to $\tilde{C} \in S^{n+1}$. 
Proof. Suppose that the constraint system (1) yields duality with respect to $C \in S^{n}$ and let $d \in R$. We show that the constraint system (12) yields duality with respect to $\tilde{C}$.

Since (SDSIP1) is a homogeneous system, we need only show that if its dual problem is inconsistent, (SDSIP1) has a value of $-\infty$ with respect to $\tilde{C}$.

Assume that (14) and (15) are inconsistent. Note that (14) is the dual constraint system (SDSIP), which has duality by hypothesis. If (SDSIP) and (14) are consistent, then,

$$
\begin{gathered}
\inf \{C \bullet X \mid A(t) \bullet X \geq b(t), t \in B, X \succeq 0\}= \\
\sup \left\{\sum_{t \in B} y(t) b(t) \mid \sum_{t \in B} y(t) A(t)+Z=C, y \in \Lambda_{B}, Z \succeq 0\right\} .
\end{gathered}
$$

Thus, it follows that at least one of these three conditions holds: (i) the constraint (14) is inconsistent; (ii) the constraint (14) is consistent, but the problem (SDSIP) is inconsistent; (iii) the constraint (14) and (SDSIP) are consistent, but

$$
d>\inf \{C \bullet X \mid A(t) \bullet X \geq b(t), t \in B, X \succeq 0\} .
$$

If (i) holds, then the problem (SDSIP) is unbounded in value by hypothesis. Thus, for any number $n$ however large, there exists $X^{(n)} \succeq 0$ such that

$$
\begin{aligned}
A(t) \bullet X^{(n)} & \geq b(t), \quad \forall t \in B, \\
C \bullet X^{(n)} & <d-n .
\end{aligned}
$$

Set

$$
\tilde{X}^{(n)}=\left(\begin{array}{cc}
X^{(n)} & \mathbf{0} \\
\mathbf{0}^{T} & 1
\end{array}\right) \text { and } \mathbf{0} \in R^{n} .
$$

It follows from $X^{(n)}$ that $\tilde{X}^{(n)}$ is a positive semidefinite matrix. Thus, we have that

$$
\begin{aligned}
\tilde{A}(t) \bullet \tilde{X}^{(n)} & \geq 0, \forall t \in B, \\
\tilde{C} \bullet \tilde{X}^{(n)} & =C \bullet X^{(n)}-d<-n .
\end{aligned}
$$

Therefore, (SDSIP1) has value $-\infty$.

If (ii) holds, then we have that (SDSIP) is inconsistent and (DSDSIP) is consistent. Since (SDSIP) has duality with respect to $C$, for any $n$, there exists a solution 
$y \in \Lambda_{B}$ for (DSDSIP) with $\sum_{t \in B} y(t) b(t) \geq n$. Then, (14) and (15) are consistent for any $d \in R$, which contradict the assumption that the dual problem is inconsistent.

If (iii) holds, then there exists a point $\bar{X} \succeq 0$ with

$$
A(t) \bullet \bar{X} \geq b(t), \forall t \in B
$$

and

$$
d>C \bullet \bar{X} .
$$

Set

$$
\tilde{X}=\left(\begin{array}{cc}
\bar{X} & \mathbf{0} \\
\mathbf{0}^{T} & 1
\end{array}\right) \text { and } \mathbf{0} \in R^{n}
$$

Then,

$$
\begin{aligned}
\tilde{A}(t) \bullet \tilde{X} & \geq 0, \quad \forall t \in B, \\
\tilde{C} \bullet \tilde{X} & <0 .
\end{aligned}
$$

Therefore, by Lemma 3.1, (SDSIP1) has value $-\infty$. We have proved the necessity of this lemma.

To prove the sufficiency of the lemma, we suppose that, for all $d \in R$ and $C \in S^{n}$, (SDSIP1) yields duality with respect to $\tilde{C}$. We need to prove that (SDSIP) yields duality with respect to $C$.

If (SDSIP) is inconsistent, then, there is only zero to solve (SDSIP1). By the definition of duality, (14) and (15) are consistent for any $d \in R$. Take $d=n$. Thus, there exists $y^{(n)} \in \Lambda_{B}$ such that

$$
\sum_{t \in B} y^{(n)}(t) A(t)+Z=C, \quad Z \succeq 0, \quad y^{(n)} \in \Lambda_{B},
$$

and

$$
\sum_{t \in B} y^{(n)}(t) b(t) \geq n, \quad y^{(n)} \in \Lambda_{B} .
$$

Therefore, clause (ii) of the Definition 2.1 holds.

If (SDSIP) is consistent, then there are two cases: 
(a) (SDSIP) is unbounded in value. We show that in this case (DSDSIP) must be inconsistent. If (DSDSIP) is consistent, then take any feasible solutions $X$ and $(y, Z)$ for (SDSIP) and (DSDSIP), respectively. Thus,

$$
C \bullet X \geq Z \bullet X+\sum_{t \in B} y(t) b(t)
$$

Since $X$ and $Z$ are positive semidefinite matrices, $Z \bullet X \geq 0$. It follows that

$$
C \bullet X \geq \sum_{t \in B} y(t) b(t)
$$

which contradicts unboundedness for (SDSIP). Thus, (DSDSIP) is inconsistent.

(b) (SDSIP) is bounded in value. Let $z_{0}=\inf \{C \bullet X \mid A(t) \bullet X \geq b(t), \quad t \in B\}$. We first show that (SDSIP1) for $d=z_{0}$ cannot be unbounded in value.

If (SDSIP1) is unbounded in value, then by Lemma 3.1, there is a solution to

$$
\begin{aligned}
\tilde{A}(t) \bullet \tilde{X} & \geq 0, \quad t \in B, \tilde{X} \succeq 0, \\
\tilde{C} \bullet \tilde{X} & <0 .
\end{aligned}
$$

Suppose $\tilde{X}=\left(\begin{array}{cc}X^{*} & x \\ x^{T} & x_{n+1}\end{array}\right)$, where $x \in R^{n}, X^{*} \in S^{n}$ and $X^{*} \succeq 0$. Take $\bar{X}=$ $\left(\begin{array}{cc}X^{*} & \mathbf{0} \\ \mathbf{0}^{T} & x_{n+1}\end{array}\right)$, where $\mathbf{0} \in R^{n}$. Obviously, $\bar{X}$ is positive semidefinite and satisfy:

$$
\begin{aligned}
\tilde{A}(t) \bullet \bar{X} & \geq 0, \quad t \in B, \bar{X} \succeq 0, \\
\tilde{C} \bullet \bar{X} & <0 .
\end{aligned}
$$

If $x_{n+1}>0$, then we may assume $x_{n+1}=1$ by homogeneity, and we have

$$
\begin{gathered}
A(t) \bullet X^{*} \geq b(t), \quad t \in B, X^{*} \succeq 0, \\
C \bullet X^{*} \quad<d,
\end{gathered}
$$

which is a contradiction to the definition of $d$.

If $x_{n+1}=0$, then, we have

$$
\begin{aligned}
A(t) \bullet X^{*} & \geq 0, \quad t \in B, X^{*} \succeq 0, \\
C \bullet X^{*} & <0 .
\end{aligned}
$$


Let $X_{0}$ be a feasible solution for (SDSIP). Then, for any $\lambda \geq 0, X_{0}+\lambda X^{*}$ is a solution of (SDSIP). Thus, we have that $C \bullet\left(X_{0}+\lambda X^{*}\right)=C \bullet X_{0}+\lambda C \bullet X^{*}<z_{0}$, for large $\lambda>0$. This is a contradiction to the definition of $z_{0}$. Then, (SDSIP1) is bounded in value for $d=z_{0}$. Since (SDSIP1) has duality, there exists a solution $(y, Z)$ satisfying (14) and (15). By (14) and (15), we have

$$
\begin{aligned}
& \sum_{t \in B} y(t) A(t)+Z=C, \quad y \in \Lambda_{B}, \\
& \sum_{t \in B} y(t) b(t) \geq d, y \in \Lambda_{B} .
\end{aligned}
$$

It follows from $d=z_{0}$ that

$$
\begin{aligned}
& \sup \left\{\sum_{t \in B} y(t) b(t) \mid \sum_{t \in B} y(t) A(t)+Z=C, \quad y \in \Lambda_{B}, Z \succeq 0\right\} \\
& \geq \inf \{C \bullet X \mid A(t) \bullet X \geq b(t), \quad t \in B\} .
\end{aligned}
$$

From (14) and (1), we have

$$
\begin{aligned}
& \sup \left\{\sum_{t \in B} y(t) b(t) \mid \sum_{t \in B} y(t) A(t)+Z=C, \quad y \in \Lambda_{B}, Z \succeq 0\right\} \\
& \leq \inf \{C \bullet X \mid A(t) \bullet X \geq b(t), \quad t \in B\}
\end{aligned}
$$

i.e., the optimal value of (SDSIP) is equal to that of (DSDSIP). So (SDSIP) yields duality with respect to $C$.

From Lemma 4.1 and Definition 2.1, we can get the following corollary.

Corollary 4.1 (SDSIP) yields uniform duality if and only if, for any $d \in R$ and $C \in S^{n}$, the constraint system (12) yields duality with respect to $\tilde{C} \in S^{n+1}$.

Theorem 4.1 (SDSIP) yields uniform duality if and only if cone $(\tilde{W})+\tilde{K}$ is a closed set, where

$$
\tilde{W}=\left\{\tilde{A}(t) \in S^{n+1} \mid t \in B\right\}, \operatorname{cone}(\tilde{W})=\left\{\sum_{t \in B} y(t) \tilde{A}(t) \mid y \in \Lambda_{B}\right\}
$$

and

$$
\tilde{K}=\left\{\left(\begin{array}{cc}
K & \mathbf{0} \\
\mathbf{0}^{T} & k
\end{array}\right) \in S^{n+1} \mid K \in S^{n}, K \succeq 0 \text { and } k \in R^{+}\right\}
$$


Proof. By Corollary 4.1, (SDSIP) yields uniform duality if and only if, for any $d \in R$ and $C \in S^{n}$, the constraint system (12) yields duality with respect to each $\tilde{C} \in S^{n+1}$. By Proposition 3.1, for any $d \in R$ and $C \in S^{n}$, the constraint system (12) yields duality with respect to each $\tilde{C} \in S^{n+1}$ if and only if $\operatorname{cone}(\tilde{W})+\tilde{K}$ is a closed set. Then, the conclusion follows readily.

\section{The Slater Condition for (SDSIP)}

It is well known that the Slater condition is a very important constraint qualification in the study of dual problem for (SDP). If a Slater condition holds, there is a zero duality gap between (SDP) and its dual. We naturally ask whether a similar result also holds for problem (SDSIP). We next discuss the problem. In fact, we establish that a Slater condition, together with a suitable compact condition, is sufficient for (SDSIP) to have uniform duality.

Now suppose that there exists an $X_{0} \succeq 0$ such that

$$
A(t) \bullet X_{0}>b(t), \quad t \in B .
$$

i.e., Slater condition holds for (SDSIP).

Lemma 5.1 Suppose $\tilde{A}(t)(t \in B)$ is generalized canonically closed and there exists $X_{0} \succeq 0$ such that (16) holds. Then, cone $(\tilde{W})+\tilde{K}$ is a closed set.

Proof. Suppose

$$
X^{*}=\left(\begin{array}{ll}
X_{0} & \mathbf{0} \\
\mathbf{0}^{T} & 1
\end{array}\right) \text {. }
$$

By (16), we have

$$
\tilde{A}(t) \bullet X^{*}>0, \quad t \in B .
$$

Thus, $\tilde{A}(t) \neq 0, \quad t \in B$. Since $\tilde{A}(t)(t \in B)$ is generalized canonically closed, there exists a positive constant set $\{k(t) \mid t \in B\}$ such that $V=\{k(t) \tilde{A}(t) \mid t \in B\}$ is a compact set. Then, the set $Q=\operatorname{conv}(V)$ is a compact set. Obviously, $\operatorname{cone}(V)=$ $\operatorname{cone}(\tilde{W})$. Thus, $\operatorname{cone}(\tilde{W})+(\tilde{K})$ is closed if and only if cone $(V)+\tilde{K}$ is closed. To 
show that $\operatorname{cone}(V)+\tilde{K}$ is closed, it suffices to show that $X \notin \operatorname{cone}(V)+\tilde{K}$ implies $X \notin \operatorname{cl}(\operatorname{cone}(V)+\tilde{K})$.

Suppose $X \notin \operatorname{cone}(V)+\tilde{K}$. Whenever $\lambda>0$ and $D \in \tilde{K}$, we must have

$$
\lambda X-D \notin Q .
$$

By (17), we have that

$$
U \bullet X^{*}>0, \quad \forall U \in Q,
$$

and hence

$$
0 \notin Q
$$

Set:

$$
M=\{\lambda X \mid \lambda \geq 0\}-\tilde{K} .
$$

Now, we prove that $M$ is a closed convex set. Take any sequence $\left\{Z_{n}\right\} \subset M$ with limiting point $Z_{0}$. Then, there exist $\lambda_{n}>0$ and $D_{n} \in \tilde{K}$ such that

$$
Z_{n}=\lambda_{n} X-D_{n}
$$

If sequence $\left\{\lambda_{n}\right\}$ has the converging subsequence, then, $Z_{0} \in M$.

If sequence $\left\{\lambda_{n}\right\}$ does not have the converging subsequence, we can assume that $\lambda_{n} \rightarrow+\infty$. Then,

$$
Z_{n} / \lambda_{n}=X-\frac{1}{\lambda_{n}} D_{n} \rightarrow 0,(n \rightarrow+\infty) .
$$

Thus, sequence $\left\{\frac{1}{\lambda_{n}} D_{n}\right\}$ is a converging one and

$$
\frac{1}{\lambda_{n}} D_{n} \rightarrow X,(n \rightarrow+\infty) .
$$

By the closedness of $\tilde{K}$, we have $X \in \tilde{K}$, which contradicts $X \notin \operatorname{cone}(V)+\tilde{K}$. Then, $M$ is a closed set. Obviously, $M$ is a convex set. Therefore, $M$ is a closed convex set.

It follows from (18) and (19) that the compact set $Q$ is entirely disjoint from the closed convex set $M=\{\lambda X \mid \lambda \geq 0\}-\tilde{K}$. By the separation theorem and the definitions of $\tilde{K}$ and $Q$, there exists a matrix $\bar{X}=\left(\begin{array}{cc}X_{1} & 0 \\ 0 & x\end{array}\right) \in S^{n+1}$, such that

$$
\beta=\inf _{U \in Q} U \bullet \bar{X}>\sup _{\lambda>0, D \in \tilde{K}}(\lambda X-D) \bullet \bar{X},
$$


where $X_{1} \in S^{n}$ and $x \in R$.

We claim that $\bar{X}$ is a positive semidefinite matrix. If not, there exists a matrix $D_{0} \in \tilde{K}$ such that

$$
D_{0} \bullet \bar{X}<0
$$

Then, the supremum in (20) is $+\infty$ and this contradicts (20).

Moreover, we have that

$$
\sup _{\lambda>0} \lambda X \bullet \bar{X}=0 .
$$

Otherwise we have $\sup _{\lambda>0} \lambda X \bullet \bar{X}>0$, thus the supremum in (20) is $+\infty$, by the arbitrariness of $\lambda$. This contradicts (20).

We reach a contradiction to $X \in \operatorname{cl}(\operatorname{cone}(V)+\tilde{K})$ as follows. Suppose that $X^{(n)} \rightarrow X$ with all $X^{(n)} \in \operatorname{cone}(V)+\tilde{K}$. Write

$$
\begin{aligned}
X^{(n)} & =\sum_{t \in B} y^{(n)}(t) k(t) \tilde{A}(t)+D_{n}, \quad y^{(n)} \in \Lambda_{B}, \\
\delta_{n} & =\sum_{t \in B} y^{(n)}(t) \geq 0,
\end{aligned}
$$

where $D_{n} \in \tilde{K}$.

Suppose $\lim \inf _{n \rightarrow+\infty} \delta_{n}=0$. Since $V=\{k(t) \tilde{A}(t) \mid t \in B\}$ is a compact set, we have that $\sum_{t \in B} y^{(n)}(t) k(t) \tilde{A}(t) \rightarrow 0$. Hence, $X \in \tilde{K} \subset$ cone $(V)+\tilde{K}$, which contradicts the assumption on $X$. Without loss of generality, we can assume that there is a scalar $\epsilon>0$ with $\delta_{n} \geq \epsilon, \forall n$. We have, by (22),

$$
\begin{aligned}
X \bullet \bar{X} & =\lim _{n \rightarrow+\infty} X^{(n)} \bullet \bar{X} \\
& =\lim _{n \rightarrow+\infty}\left(\sum_{t \in B} y^{(n)}(t) k(t) \tilde{A}(t)\right) \bullet \bar{X}+D_{n} \bullet \bar{X} \\
& \geq \lim _{n \rightarrow+\infty} \delta_{n}\left(\sum_{t \in B} \frac{y^{(n)}(t)}{\delta_{n}}(k(t) \tilde{A}(t))\right) \bullet \bar{X} \\
& \geq \epsilon \liminf _{n \rightarrow+\infty}\left(\sum_{t \in B} \frac{y^{(n)}(t)}{\delta_{n}}(k(t) \tilde{A}(t))\right) \bullet \bar{X} \\
& \geq \epsilon \beta>0
\end{aligned}
$$

since $\sum_{t \in B} \frac{y^{(n)}(t)}{\delta_{n}} k(t) \tilde{A}(t) \in Q$. But, by $(21), X \bullet \bar{X} \leq 0$. This is a contradiction. Thus, this completes the proof. 
Theorem 5.1 Suppose that $\tilde{A}(t)(t \in B)$ is generalized canonically closed and that there exists $X_{0} \succeq 0$ such that (16) holds. Then, (SDSIP) yields uniform duality.

Proof. By Lemma 5.1, we have that cone $(\tilde{W})+\tilde{K}$ is a closed convex set. It follows from Theorem 4.1 that (SDSIP) yields uniform duality. Thus, this completes the proof.

Corollary 5.1 Suppose that $\tilde{W}$ is a compact set and that there exists $X_{0} \succeq 0$ such that (16) holds. Then, (SDSIP) yields uniform duality.

Proof. If $\tilde{W}$ is a compact set, then, $\tilde{A}(t)(t \in B)$ is generalized canonically closed. Thus, it follows from Theorem 5.1 that the corollary holds.

If $b(t)=0(t \in B)$, then, (SDSIP) becomes homogeneous system $\left(\operatorname{SDSIP}_{h}\right)$ and the Slater condition (16) becomes: there exists an $X_{0} \succeq 0$ such that

$$
A(t) \bullet X_{0}>0, \quad t \in B
$$

Combining Theorem 5.1 and Remark 2.2, we get the following corollary.

Corollary 5.2 Suppose that $A(t)(t \in B)$ is generalized canonically closed and that there exists $X_{0} \succeq 0$ such that (24) holds. Then, (SDSIP ${ }_{h}$ ) yields uniform duality.

The following three examples illustrate Corollary 5.2.

Example 3.1 (continued) We know that $W=\left\{\left(\begin{array}{cc}-\sin (t) & 0 \\ 0 & \cos (t)\end{array}\right) \mid t \in[0, \pi / 2]\right\}$ is a compact set. However, the Slater condition (24) does not hold. We know that the primal problem does not yield uniform duality. Thus, if the Slater condition (24) is dropped, Corollary 5.2 may not hold.

Example 5.1 Suppose

$$
\begin{gathered}
a(t)=\left\{\begin{array}{cl}
0, & t=T_{1}, \\
-\frac{1}{t-T_{1}}, & t \neq T_{1},
\end{array}\right. \\
A(t)=\left(\begin{array}{cc}
a(t) & 0 \\
0 & 1
\end{array}\right), t \in\left[T_{1}, T_{2}\right]\left(T_{2}>T_{1}\right) \text { and } C=\left(\begin{array}{cc}
-2 & -1 \\
-1 & 0
\end{array}\right) .
\end{gathered}
$$


The primal problem is

$$
\begin{array}{ll}
\text { inf } & C \bullet X \\
\text { s.t } & A(t) \bullet X \geq 0, \quad t \in\left[T_{1}, T_{2}\right], \\
& X \succeq 0 .
\end{array}
$$

We have that $A(t)\left(t \in\left[T_{1}, T_{2}\right]\right)$ is not generalized canonically closed and that the feasible set of the primal problem is

$$
\mathcal{F}=\left\{\left(\begin{array}{ll}
0 & 0 \\
0 & x
\end{array}\right) \mid x \geq 0\right\}
$$

Take $X_{0}=\left(\begin{array}{ll}0 & 0 \\ 0 & 1\end{array}\right)$. We have that

$$
A(t) \bullet X_{0}>0, \quad \forall t \in\left[T_{1}, T_{2}\right]
$$

i.e., the Slater condition (24) holds.

The primal problem has an optimal solution $X^{*}=\left(\begin{array}{ll}0 & 0 \\ 0 & 0\end{array}\right)$, and the optimal value is zero.

The dual problem of the primal problem is

$$
\begin{array}{ll}
\text { sup } & 0 \\
\text { s.t } & \sum_{t \in\left[T_{1}, T_{2}\right]} y(t) A(t)+Z=C, y \in \Lambda_{\left[T_{1}, T_{2}\right]}, \\
& Z \succeq 0 .
\end{array}
$$

However, the dual problem has no feasible solution. i.e., the dual problem is inconsistent. Thus, the primal problem does not yield uniform duality. This shows that if the generalized canonically closedness of $A(t)(t \in B)$ is dropped, Corollary 5.2 may not hold.

Example 3.2 (continued) We know that $W=\{A(t) \mid t \in[1,2]\}$ is a compact set. Take $X_{0}=\left(\begin{array}{cc}1 & 0 \\ 0 & 1\end{array}\right)$. Then, $X_{0} \succeq 0$ and

$$
A(t) \bullet X_{0}>0, \quad t \in[1,2]
$$


Thus, the Slater condition (24) holds. It follows from Corollary 5.2 that the primal problem yields uniform duality.

From Theorem 5.1 and Definition 2.1, we obtain the following result.

Corollary 5.3 Suppose that $\tilde{A}(t)(t \in B)$ is generalized canonically closed and there exists $X_{0} \succeq 0$ such that (16) holds, and suppose that (SDSIP) has finite infimum M. Then, (DSDSIP) is also consistent and has a finite supremum $M^{\prime}$. Moreover, $M^{\prime}=M$ and the value is attained.

Next we consider two special cases of (SDSIP): (SDP) and (SILP). If $B=\{1,2, \cdots, m\}$, (SDSIP) problem becomes the general (SDP) problem:

$$
\begin{array}{ll}
\text { inf } & C \bullet X \\
\text { S.t. } & A_{i} \bullet X \geq b_{i}, \quad i=1,2, \cdots, m, \\
& X \succeq 0 .
\end{array}
$$

(DSDSIP) problem becomes the problem (DSDP):

$$
\begin{array}{ll}
\sup & \sum_{i=1}^{m} b_{i} y_{i} \\
\text { s.t } & \sum_{i=1}^{m} y_{i} A_{i}+Z=C, y_{i} \geq 0, i=1,2, \cdots, m \\
& Z \succeq 0
\end{array}
$$

Obviously, $\tilde{A}_{i}(i=1,2, \cdots, m)$ always is generalized canonically closed. Thus, by Corollary 5.3, we can get the duality theorem for (SDP).

Theorem 5.2 Suppose that there exists $X_{0} \succeq 0$ such that (16) holds and that (SDP) problem has a finite infimum $M$. Then, its dual problem (DSDP) is also consistent and has a finite supremum $M^{\prime}$. Moreover, $M^{\prime}=M$ and the value is attained.

If

$$
C=\left(\begin{array}{ccc}
c_{1} & & 0 \\
& \ddots & \\
0 & & c_{n}
\end{array}\right), A(t)=\left(\begin{array}{ccc}
a_{1}(t) & & 0 \\
& \ddots & \\
0 & & a_{n}(t)
\end{array}\right), \forall t \in B
$$


and $x=\left(x_{1}, x_{2}, \cdots, x_{n}\right)^{T}$, (SDSIP) problem is reduced to a semi-infinite linear programming problem (SILP):

$$
\begin{array}{ll}
\text { inf } & c^{T} x \\
\text { s.t } & (a(t))^{T} x \geq b(t), \quad t \in B, \\
& x \geq 0,
\end{array}
$$

where $c=\left(c_{1}, c_{2}, \cdots, c_{n}\right)^{T}$ and $a(t)=\left(a_{1}(t), a_{2}(t), \cdots, a_{n}(t)\right)^{T}$. (DSDSIP) problem is reduced to the problem (DSILP):

$$
\begin{array}{ll}
\sup & \sum_{t \in B} b(t) y(t) \\
\text { s.t } & \sum_{t \in B} y(t) a(t) \leq c, \\
& y \in \Lambda_{B} .
\end{array}
$$

Thus, by Corollary 5.3, we can get the duality theorem for (SILP).

Theorem 5.3 (See [2]) Suppose $\left(a_{1}(t), a_{2}(t), \cdots, a_{n}(t), b(t)\right)^{T}(t \in B)$ is canonically closed and that there exists $x_{0} \succeq 0$ such that

$$
a(t)^{T} x_{0}>b(t), t \in B
$$

holds. Let (SILP) problem have finite infimum $M$. Then, its dual problem (DSILP) is also consistent and has a finite supremum $M^{\prime}$. Moreover, $M^{\prime}=M$ and the value is attained.

Remark 2.3 Theorem 5.3 is the same as the duality theorem on pp.127 of [2]. Thus, Corollary 5.3 is a generalization of the duality theorem in [2].

\section{References}

[1] Charnes, A., Cooper, W. W. and Kortanek, K.(1962), Duality in Semi-Infinite Programs and Some Works of Haar and Caratheodory, Management Sciences 9, 209-229. 
[2] Duffin, R. J., Jeroslow, R. G. and Karlovitz, L. A.(1983), Duality in Semiinfinite Linear Programming, in Fiacco, A.V. and Kortanek, K. O. (eds.), Semi-Infinite Programming and Applications, Lecture Notes in Economics and Mathematical Systems 215, Springer-Verlag Berlin Heidelberg New York Tokyo, $50-62$.

[3] Duffin, R. J. and Karlovitz, L. A.(1965), An Infinite Linear Program with a Duality Gap, Management Science 12, 122-134.

[4] Hettich, R. and Kortanek, K.O.(1993), Semi-Infinite Programming: Theory, Methods, and Applications, SIAM Review 35, 380-429.

[5] Jeyakumar, V. and Gwinner, J.(1991), Inequality Systems and Optimization, Journal of Mathematical Analysis and Applications 159, 51-71.

[6] Kelley, J. L. and Namioka, I.(1963), Linear Topological Spaces, Springer-Verlag, New York.

[7] Lieven Vandenberghe and Stephen Boyd (1996), Semidefinite Programming, SIAM Review 38, 49-95.

[8] Lieven Vandenberghe and Stephen Boyd (1998), Connections between Semiinfinite and Semidefinite Programming, in Rembert Reemtsen and Jan-J. Ruchmann (eds.), Semi-Infinite Programming, Nonconvex Optimization and Its Applications 25, Kluwer Academic Publishers Boston/Dordreccht/London, 277294.

[9] Nayakkankuppam, Madhu V. and Overton, Michael L.(1999), Conditioning of Semidefinite Programs, Mathematical Programming, Series A 85, 525-540.

[10] Pardalos, P. M.(1991), Quadratic Programming with One Negative Eigenvalue Is NP-Hard, Journal of Global Optimization 1, 15-22.

[11] Ramana, M. V.(1997), An Exact Duality Theory for Semidefinite Programming and Its Complexity Implications, Mathematical Programming 77, 129-162. 
[12] Ramana, M. V., Tuncel, L. and Wolkowicz, H.(1997), Strong Duality for Semidefinite Programming, SIAM Journal on Optimization 7, 641-662.

[13] Reemtsen, R. and Ruckmann, J.J. (1998), Semi-Infinite Programming, Kluwer Academic Publisher.

[14] Stoer, J. and Witzgall, C.(1970), Convexity and Optimization in Finite Dimensions: I, Springer-Verlag, New York.

[15] Wolkowicz, H., Saigal, R. and Vandenberghe, L.(2000), Handbook of Semidefinite Programming Theory, Algorithms, and Applications, Kluwer Academic Publishers. 